\title{
The Inter-temporal Securities Portfolio Model Based on Inter Programming
}

\author{
Dong Yu \\ Dalian Maritime University \\ Dalian, China \\ e-mail:Garden_1@163.com \\ Yuzhen Lu \\ Dalian Maritime University \\ Dalian, China \\ e-mail: dlyuzhen@dlmu.edu.cn
}

\author{
Qing Li \\ Dalian Maritime University \\ Dalian, China \\ e-mail: 912809839@qq.com \\ Min Zhang* \\ Dalian Maritime University \\ Dalian, China \\ e-mail: zhangmin071@ sina.com
}

\begin{abstract}
In order to satisfy the inter-temporal securities investors, we discuss the inter-temporal securities portfolio model with adding transaction fees in this paper. Meanwhile, for the purpose of improving the practicality of the model, we take the transaction fees, the trading volume and the minimum trading unit into account in founding the model. We divide the main content of this paper into three parts. First, Obtaining the yield and variance buy least squares in a specific period of time. Then, founding the inter-temporal securities portfolio model with adding transaction fees. Finally, obtaining the integral solution of the model. The proposed of this model provide a valuable reference standard for inter-temporal securities investors.
\end{abstract}

Keywords-inter-temporal; Portfolio selection; Integer programming; Least square method; Transaction fees.

\section{INTRODUCTION}

Investors are getting more and more investment opportunities with the rapid development of capital market. For the investors, How to decide the return and risk of portfolio investment and how to balance the two indexes in the asset allocation are the two core problems in the process of investment. Since the mean-variance model proposed by Markowitzin 1952[1], In order to get the optimal solution of mean-variance model, many scholars spend much time in studing it. They used different kind of algorithms for solving the mean-variance model.Such as the Genetic Algorithm[2], Particle Swarm Algorithm[3], Neural network[4], and so on.It is difficult to get the best result. In order to make the solving process more accurate and simple, Huang Xiaoxia [5] and Liu Jianjun [6] study portfolio model base on uncertainty theory [7].They take the yield of each security as a random variable which obey uncertainty distribution, they also assume the securities are independent from each other.Then they can change the nonlinear mean-variance model into linear programming model, which improved the calculation speed and accuracy.In fact, they have ignored the investment restrictions which are short selling, transaction fees, the trading volume and the minimum trading unit in founding the model. It will greatly reduce the usefulness of the model if they don't take the investment restrictions into account. Moreover, for the inter-temporal securities investors, they lack a good reference model. In order to overcome this phenomenon.This article focuses on the inter-temporal securities portfolio model base on the uncertainty theory.If we want to know the investment amount of each security we must get the yield and variance of each security. In this paper we take the yields of securities as a random variable which obey uncertainty distribution. So we use the least square method to estimate the yield and variance of each security, according to the predicted values of many experts for the same security[8][9][10]. We take cumulative variance of multiple investment as the total risk in the inter-temporal securities portfolio model. The better result provide a valuable reference standard for investors.Fundamentals of Uncertainty Theory

Definition 1: Let $\Gamma$ is a nonempty set, and $L$ a $\sigma$-algebra over $\Gamma$.Each element $\Lambda \in L$ is called an event, A set function $M\{\Lambda\}$ is called an uncertain measure if it satisfies the following three axioms:

(i) (Normality) $M\{\Lambda\}=1$.

(ii) (Self-duality) For any event $\Lambda$,we have $M\{\Lambda\}+M\left\{\Lambda^{c}\right\}=1$.here $\Lambda^{c}$ is the complement of $\Lambda$.

(iii) ( Countable subadditivity) For every countable sequence of events $\left\{\Lambda_{i}\right\}$,

we have $M\left\{\bigcup_{i=1}^{\infty} \Lambda_{i}\right\} \leq \sum_{i=1}^{\infty} M\left\{\Lambda_{i}\right\}$.

Definition 2: Let $\Gamma$ is a nonempty set, and $L$ a $\sigma$-algebra over $\Gamma, M$ is an uncertain measure. Then the triplet $(\Gamma, L, M)$ is called an uncertainty space.

Theorem 1: Any uncertain measure $M$ is increasing, for any events $\Lambda_{1} \subset \Lambda_{2}$, we have $M\left\{\Lambda_{1}\right\} \leq M\left\{\Lambda_{2}\right\}$.

Definition 3: An uncertain variable is a measurable function $\mathrm{n}$ from an uncertainty space $(\Gamma, L, M)$ to the set of real numbers, for any Borel set B of real numbers, the $\operatorname{set}\{\xi \in B\}=\{\gamma \in \Gamma \mid \xi(\gamma) \in B\}$ is an event. 
Definition 4: For any real number $x$, The uncertainty distribution $\Phi: \mathfrak{R} \rightarrow[0,1]$ of an uncertain variable $\xi$ is defined by $\Phi(x)=M\{\xi \leq x\}$.

For example, by a normal uncertain variable $\xi$, we mean the variable that has the following normal uncertainty distribution

$$
\Phi(t)=\left(1+\exp \left(\frac{\pi(\mu-t)}{\sqrt{3} \sigma}\right)\right)^{-1} \quad, \quad t \in \mathfrak{R}
$$

(1)

Where $\mu$ and $\sigma$ are real numbers and $\sigma>0$.For convenience, it is denoted in the paper by $\xi \sim N(\mu, \sigma)$. The inverse distribution of $\xi$ is as follows:

$$
\Phi^{-1}(\alpha)=\mu+\frac{\sqrt{3} \sigma}{\pi} \ln \frac{\alpha}{1-\alpha} . \alpha \in[0,1]
$$

We call the uncertain variable is a lognormal uncertain variable if it has the following lognormal uncertainty distribution

$$
\Phi(t)=\left(1+\exp \left(\frac{\pi(\mu-\ln t)}{\sqrt{3} \sigma}\right)\right)^{-1} \quad t \in \mathfrak{R}
$$

where $\mu$ and $\sigma$ are real numbers and $\sigma>0$,For convenience, it is denoted in the paper by

$\xi \sim \operatorname{LOGN}(\mu, \sigma)$. The inverse distribution of $\xi$ is as follows:

$$
\Phi^{-1}(\alpha)=e^{\mu+\frac{\sqrt{3} \sigma}{\pi} \ln \frac{\alpha}{1-\alpha} .} \quad \alpha \in \quad[0]
$$

When the uncertain variables $\xi_{1}, \xi_{2}, \cdots, \xi_{n}$ are represented by uncertainty distributions, the operational law can be expressed as follows:

Theorem 2: Let $\xi$ be an uncertain variable with continuous uncertainty distribution $\Phi$, and let $\mathrm{f}$ be a strictly increasing function. Then the uncertainty distribution $\psi$ of $f(\xi)$ can be obtained via

$$
\psi(t)=\Phi\left(f^{-1}(t)\right)
$$

which can also be expressed by

$$
\psi^{-1}(\alpha)=f\left(\Phi^{-1}(\alpha)\right), 0<\alpha<1 \text {. }
$$

\section{THE ESTIMATED OF YIELD AND VARIANCE}

In order to obtain the uncertainty distribution of the yield and variance of each security. First, we ask $m$ economists for questionnaire survey, from the survey we can get the predicted yield and variance of $n$ securities which are given by $m$ economists, and they also predict the unit price of the securities. Assuming the economists who accepted questionnaire survey have the same authority in the stock market, and their prediction are not effected by each other.

To illustrate the solving process, here we take one securities as an example. Let $t_{i}$ as the yield of the securities, let $\alpha_{i j}$ as the value of the distribution function, where $\alpha_{i j}$ is given by the $j$-th economist when the yield of the security is $t_{i} . \quad i=1,2, \cdots, k, j=1,2, \cdots, m$. Since the distribution function $\Phi(x)$ is an increasing function, if $t_{1}<t_{2}<\cdots<t_{k}$, we have $0 \leq \alpha_{1 j} \leq \alpha_{2 j} \leq \cdots \alpha_{k j} \leq 1$. And we know that there are $m$ predicted values for each $t_{i}$.Here we assume the economists who accepted questionnaire survey have the same authority in stock market, so we take the reliability of the predicted values as the same. Therefore, we take the average value of $m$ economists as the final result of $\Phi\left(t_{i}\right)$, it can be expressed as follows:

$$
\Phi\left(t_{i}\right)=\alpha_{i}=\frac{1}{m} \sum_{j=1}^{m} \alpha_{i j} \cdot i=1,2, \cdots, k
$$

From (7) we know that $0 \leq \alpha_{1} \leq \alpha_{2} \leq \cdots \alpha_{k} \leq 1$.Here we assume the yield of the securities obey normal distribution $N(\mu, \sigma)$.We will use the least squares method to estimate the parameters $\mu$ and $\delta$.It means $\mu$ and $\delta$ should satisfy next conditions:

$$
\begin{aligned}
& \min \sum_{i=1}^{k}\left(\Phi\left(t_{i} \mid \mu, \delta\right)-\alpha_{i}\right)^{2} \\
& \text { or } \quad \min \sum_{i=1}^{k}\left(\Phi^{-1}\left(\alpha_{i} \mid \mu, \delta\right)-t_{i}\right)^{2}
\end{aligned}
$$

where

$\Phi\left(t_{i} \mid \mu, \delta\right)=\left(1+\exp \left(\frac{\pi\left(\mu-t_{i}\right)}{\sqrt{3} \delta}\right)\right)^{-1}$

$\Phi^{-1}\left(\alpha_{i} \mid \mu, \delta\right)=\mu+\frac{\sqrt{3} \delta}{\pi} \ln \frac{\alpha_{i}}{1-\alpha_{i}}$

We can obtain the estimated value of $\mu$ and $\delta$ as follows:

$$
\left\{\begin{array}{l}
\hat{\mu}=\bar{t}-\frac{\sqrt{3} \hat{\delta}}{k \pi} \sum_{i=1}^{k} \ln \frac{\alpha_{i}}{1-\alpha_{i}} \\
\hat{\delta}=\frac{\sqrt{3} \pi k}{3} \frac{t \sum_{i=1}^{k} \ln \frac{\alpha_{i}}{1-\alpha_{i}}-\sum_{i=1}^{k} t_{i} \ln \frac{\alpha_{i}}{1-\alpha_{i}}-}{\left(\sum_{i=1}^{k} \ln \frac{\alpha_{i}}{1-\alpha_{i}}\right)^{2}-n\left(\sum_{i=1}^{k} \ln \frac{\alpha_{i}}{1-\alpha_{i}}\right)^{2}}
\end{array}\right.
$$

where $\hat{\mu}$ and $\hat{\delta}$ is the estimated value of $\mu$ and $\delta$, and $\bar{t}=\frac{1}{n} \sum_{i=1}^{k} t_{i}$.

If the yields of securities obey normal distribution $\operatorname{LOGN}(\mu, \sigma)$, we also could use the same method to get the estimated value of $\mu$ and $\delta$. 


\section{INTER-TEMPORAL PORTFOLIO MODEL}

In order to improve the practicality of this model, here we set the investment periods is $n$, and the period could be day, month and year in here. Therefore, if the investment of periods is $\boldsymbol{n}$, the investors could choose $\boldsymbol{n}$ kinds of securities, the investment horizon of which are between 1 and $\boldsymbol{n}$ to invest. Since the minimum trading unt of securities is round lot which means hundred shares. And the transaction amount must be an integer. If the security of $j$-th was invested $n_{i j}$ in the period of $i$-th, then $n_{i j}$ is a nonnegative integer. Moreover, Investors need to pay transaction fees in the trading market. The transaction fees include entrust cost, commission and transfer fees.Here we take the data of shanghai securities exchange as an example. In the securities exchange entrust cost is five (yuan) per deal. The commission no more than three over one thousand, and the transfer fees payed by thousandth of the number of shares. In this paper, $C\left(n_{i j}\right)$ is the transaction fees of the $j$-th security which was invested for $n_{i j}$ in the $i$-th period.

The specific expression of $C\left(n_{i j}\right)$ is as follows:

$$
C\left(n_{i j}\right)=a_{j}+100 \beta_{j} n_{i j} p_{i}+100 \lambda_{j} n_{i j}
$$

Where $n_{i j}$ is the investment quantity of the $j$-th security in the $i$-th stage, $a_{i}$ is the entrust cost, $\beta_{j}$ is the commission, $\lambda_{j}$ is the cost coefficient of the transfer of shares, $p_{i}$ is the unit price of $i$-th securities which is the average of the prediction values which are given by $m$ economists, and $i+j \leq n+1,1 \leq i, j \leq n$.

Let $M$ is the idle funds in the initial stage of investment, then we have the following constraint:

$$
\sum_{i=1}^{n} 100 n_{i} p_{i}+C_{1} \leq M
$$

Where $C_{1}$ is the transaction fees in the first stage of investment. And we set $C_{m}$ is the transaction fees in the $m$-th stage of investment. Obviously,

$$
C_{m}=\sum_{i=1}^{n-m+1} C\left(n_{m i}\right) \text {, and we can get the following }
$$

expression from (10).

$$
\begin{aligned}
& C_{m}=\sum_{i=1}^{n-m+1} C\left(n_{m i}\right)=\sum_{i=1}^{n-m+1} a_{i}+\sum_{i=1}^{n-m+1} 100 \beta_{i} p_{i} n_{m i} \\
& +\sum_{i=1}^{n-m+1} 100 \lambda_{i} n_{m i}
\end{aligned}
$$

In the first stage, investors could sell out the securities, the investment horizon of which is one, and they could regard the recovered funds in the first stage as investment funds in the second stage, and so on. Therefore, we could derivate the constraints as follows in the $\mathrm{m}$-th stage: $\sum_{i=1}^{n-m+1} 100 n_{m i} p_{i}+C_{m} \leq \sum_{i=1}^{m-1} 100 n_{m-i, i} p_{i}\left(1+r_{i}\right)$

$1 \leq i, m \leq n$.

where $r_{i}$ is the yield of $i$-th security, the investors not only want to get the maximize profits, but also hope the risk minimization. Since the risk exist in the process of each investment. We take the cumulative risk as the total risk of the model. The risk is expressed as follows:

$$
\sum_{m=1}^{n} \sum_{i=1}^{n-m+1} 100 n_{m i} p_{i} v_{i}
$$

Now, we can get the inter-temporal securities portfolio model as follows:

$$
\begin{aligned}
& \min \sum_{m=1}^{n} \sum_{i=1}^{n-m+1} 100 n_{m i} p_{i} v_{i} \\
& \left\{\begin{array}{l}
\sum_{i=1}^{n} 100 n_{n-i+1, i} p_{i}\left(1+r_{i}\right)-\left(\sum_{i=1}^{n} 100 n_{1 i} p_{i}+C_{1}\right) \geq R_{0} \\
\sum_{i=1}^{n} 100 n_{1 i} p_{i}+C_{1} \leq M \\
\sum_{i=1}^{n-m+1} 100 n_{m i} p_{i}+C_{m} \leq \sum_{i=1}^{m-1} 100 n_{m-i, i} p_{i}\left(1+r_{i}\right) \\
i, m, n_{m i} \in N^{+}, 1 \leq i, m \leq n
\end{array}\right.
\end{aligned}
$$

Where $n_{i j}$ is the investment quantity of the $j$-th security in the $i$-th stage, $r_{i}$ is the yield of $i$-th security, $r_{i}$ is the variance of $i$-th security, $p_{i}$ is the unit price of $i$-th security, $C_{m}$ is the transaction fees in the $m$-th stage of investment, $R_{0}$ is the expected revenue, and $M$ is the idle funds.

\section{CASE ANALYSIS}

Now, an investor wants to invest one hundred thousands in four securities, and he hope the profit on less than fifty-two hundreds in four months (investment period ). The security returns are believed to be normal uncertain variables. Ten experts give their individual judgements about security returns independently. By using equation(7)-(9), the distributions of security returns are obtained and given in the follow table 1. In the table, the unit price of each security is the average value of predicted values which are given by ten economists. In this case, the equation (15) could be written as follows: 


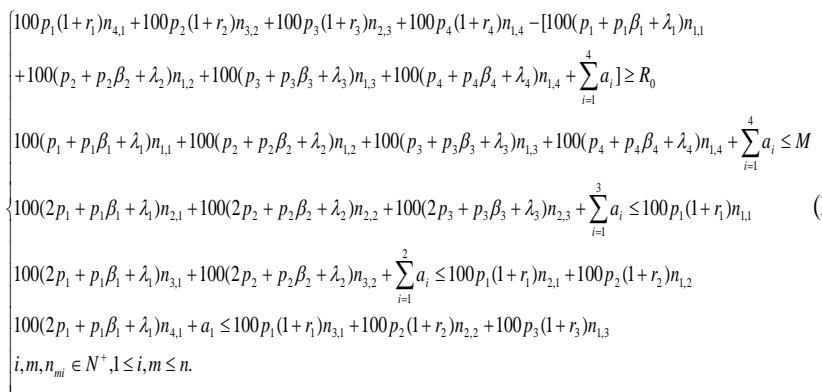

where $a_{i}=5, \beta_{i}=0.003, \lambda_{i}=0.001, i=1,2,3,4$, , $M=100000$, and the result of model (16) is $R_{0}=5200$ given in table 2 .

Table 1: Normal uncertain returns of 4 securities.

\begin{tabular}{|c|c|c|c|c|}
\hline Secunity i & 1 & 2 & 3 & 4 \\
\hline $\begin{array}{c}\text { Uncertain } \\
\text { return }\end{array}$ & $\begin{array}{c}N(0.031,0.0 \\
58)\end{array}$ & $\begin{array}{c}N(0.044,0.0 \\
73)\end{array}$ & $\begin{array}{c}N(0.051,0.0 \\
84)\end{array}$ & $\begin{array}{c}N(0.067,0.0 \\
98)\end{array}$ \\
\hline Unit price & 3.39 & 13.01 & 6.82 & 5.26 \\
\hline
\end{tabular}

Table 2: The result of each $n_{i j}$.

\begin{tabular}{|c|c|c|c|c|c|}
\hline$n_{i j}$ & $n_{11}$ & $n_{21}$ & $n_{31}$ & $n_{41}$ & $n_{12}$ \\
\hline Quantity & 2 & 1 & 0 & 1 & 0 \\
\hline$n_{i j}$ & $n_{22}$ & $n_{32}$ & $n_{13}$ & $n_{23}$ & $n_{14}$ \\
\hline Quantity & 0 & 0 & 1 & 0 & 186 \\
\hline
\end{tabular}

Obviously, the investor should buy two hands stock, one hand stock, zero hand stock, one hand stock for the first security during the four stages, bull for the second security during the rest three stages, one hand stock, zero hand stock for the third security during the third stage, and one hundred and eighty-six hands stock for the fourth security during the last stage. Which means the minimal risk is 9723.864 .

\section{CONCLUSION}

In this paper, we mainly discuss the inter-temporal securities portfolio model base on uncertainty theory. First, According to the estimated data which given by economists, we could get the yield and variance by least squares in a specific period of time. Then establish the inter-temporal securities portfolio model with transaction fees added. Finally, obtaining the integral solution of the model. The value of this subject is to help the inter-temporal securities investors how to invest in different kinds of securities. .

\section{REFERENCES}

[1] Markowitz H.:Portfolio Selection[J]. Journal of Finance.7(1),77--91 (1952)

[2] Chen Guohua, Chen Shou, Liao Xiaolian:Genetic Algorithm for fractional programming of fuzzy portfolio model[J]. Fuzzy Sets and Systems.23:163-167(2009)

[3] Liu Donghua, Gan Ruoxun,Fan Suohai,Yang Minghua: Particle swarm optimization based on predatory search for portfolio investment [J]. Computer Engineering and Applications. Web publishing time. 04.25(2012)

[4] Liu Erdong: Portfolio research base on neural network and Particle Swarm Optimization Algorithm[D]. Guangzhou, South China University of Technology(2011)
[5] Xiaoxia Huang: Mean-variance models for portfolio selection subject to experts' estimations[J]. Expert Systems with Applications. 39,5887-5893(2012)

[6] Liu Jianjun: Research on New Optimal Portfolio Selection Model with Uncertain Returns[J].Computer scienc-e.38,199-202(2011)

[7] Liu,B.Berlin: Springer-Verlag[Chapter5]. Uncertainty Theory(2nd ed.2007)

[8] Zhang Xin: Portfolio model based on linear programming[J]. Decision-Making and reference.5,68-71(2011)

[9] E. Louis, J. H. Rachel and Y. T. Larry, Precautionary effort: a new look, The journal of Risk and Insurance, vol.79, 585-590, 2012.

[10] ZHAO Lei, GAO Jianli: Impact of Accidental Expenditure on Inter-Temporal Uncertainty Decision of Household Consumption and Saving In Life-Cycle[J]. China International Conference on Insurance and Risk Management.340-361(2013) 